\title{
Combined Wavelet Video Coding and Error Control for Internet Streaming and Multicast
}

\author{
Tianli Chu \\ Department of Electrical Engineering, Texas AઐM University, College Station, TX 77843, USA \\ Email: ctl@ee.tamu.edu

\section{Zixiang Xiong} \\ Department of Electrical Engineering, Texas A\&M University, College Station, TX 77843, USA \\ Email: zx@ee.tamu.edu
}

Received 23 December 2001 and in revised form 9 September 2002

\begin{abstract}
This paper proposes an integrated approach to Internet video streaming and multicast (e.g., receiver-driven layered multicast (RLM) by McCanne) based on combined wavelet video coding and error control. We design a packetized wavelet video (PWV) coder to facilitate its integration with error control. The PWV coder produces packetized layered bitstreams that are independent among layers while being embedded within each layer. Thus, a lost packet only renders the following packets in the same layer useless. Based on the PWV coder, we search for a multilayered error-control strategy that optimally trades off source and channel coding for each layer under a given transmission rate to mitigate the effects of packet loss. While both the PWV coder and the error-control strategy are new-the former incorporates embedded wavelet video coding and packetization and the latter extends the single-layered approach for RLM by Chou et al.- the main distinction of this paper lies in the seamless integration of the two parts. Theoretical analysis shows a gain of up to $1 \mathrm{~dB}$ on a channel with $20 \%$ packet loss using our combined approach over separate designs of the source coder and the error-control mechanism. This is also substantiated by our simulations with a gain of up to $0.6 \mathrm{~dB}$. In addition, our simulations show a gain of up to $2.2 \mathrm{~dB}$ over previous results reported by Chou et al.
\end{abstract}

Keywords and phrases: wavelet video coding, error control, internet streaming, multicast, PWV.

\section{INTRODUCTION}

In recent years, we have witnessed explosive growth of the Internet. Driven by the rapid increase of bandwidth and computing power and, more importantly, the consumer's insatiable demand for multimedia content, media streaming over the Internet has quickly evolved from novelty to mainstream in multimedia communications. As the flagship application that underscores the ongoing Internet revolution, video streaming has become an important way for information distribution. For example, distance learning, telemedicine, and live webcast of music concerts and sports events are all benefiting from video streaming technology. People are already more and more dependent on this new technology in their daily lives and business. As such, Internet video streaming has attracted attention from both the industry (e.g., Microsoft and RealNetworks) and academia $[1,2,3]$.

From a schematic point of view, Internet video streaming involves video compression, Quality-of-Service (QoS) control (error control and congestion control), streaming servers, streaming protocols, and media synchronization, of which the first two components are the most important.
Compression is a must in video streaming, because full motion video requires at least $8 \mathrm{Mbps}$ bandwidth. A compression ratio of over 200:1 is needed for the transmission of video over a $56 \mathrm{kbps}$ modem connection! International standards like MPEG-4 [4] and H.263+ [5] for video compression have been developed during the past five years for the applications related to streaming media. Nowadays, commercial client players (e.g., QuickTime, Windows Media Player and RealOnePlayer) employ mostly MPEG-4 or H.263+ related technologies. In the meanwhile, corporate companies are developing new scalable video-coding technology over and beyond the MPEG-4 and H.263+ standards. For example, Microsoft chose the 3D SPIHT coder [6] as a core technology in its nextgeneration video streaming product. A key feature of the 3D SPIHT coder is that it is $100 \%$ scalable-there is no performance penalty due to scalability. This is different from MPEG-4 fine-granularity scalable (FGS) coding [7], which suffers a loss of $1-1.5 \mathrm{~dB}$ compared to single-layer MPEG-4 coding. Details on source coding-3D embedded wavelet video coding in particular-are provided in Section 2.1 . 
Another reason for our emphasis on 3D embedded wavelet video coding is that today's streaming video applications mostly use unicast. There is an increasing momentum to move towards multicast applications [8] and bring the broadcasting flavor [9] to the streaming world. Because layered source coding can be conveniently used to deal with bandwidth heterogeneity in the Internet, it is the foundation of receiver-driven layered multicast (RLM) [10], in which the sender broadcasts source and parity packets to different multicast groups after layered source coding and channel coding. Each receiver estimates its available bandwidth and accordingly subscribes to the right combination of multicast groups to optimize its video quality.

Since the Internet is the best-effort network that offers no QoS guarantee, ambient packet loss is inevitable in the Internet and the use of error-control techniques is thus necessary. The purpose of error control is to use the available transmission rate, as determined by the congestioncontrol mechanism $[11,12]$, to mitigate the effects of packet loss. Error control is generally accomplished by transmitting some amount of redundant information to compensate for the loss of some important packet. This is achieved via joint source-channel coding (JSCC) $[13,14,15]$ by finding the optimal source-redundancy mix or source-channel coding trade-off. Internet video streaming requires that packets be received within a bounded delay. Therefore, errorcontrol techniques, such as forward error correction (FEC), are often used. Unequal error protection (UEP) using ratecompatible codes was popularized by Hagenauer [16]. It can be achieved by fixing the source block length $K$ and varying the channel block length $N$ across the different source layers.

Chou et al. [17] addressed error control for RLM based on the 3D SPIHT coder [6] that encodes each group of frames (GOF) of a video sequence into an embedded bitstream. Each 3D SPIHT bitstream is uniformly divided into a series of 1000-byte source packets; parity packets are generated with Reed-Solomon (RS) style erasure codes; an iterative descent algorithm is used to compute the JSCC solution in the form of UEP for the given bandwidth and packet loss probability. Each receiver first estimates the channel condition and then follows this solution to join the multicast groups for optimal collection of source and parity packets.

While the error-control mechanism in [17] can be applied to any layered source bitstream, no interaction exists between source coding and error control. This separate design philosophy has some drawbacks. To see this, we note that 3D SPIHT packets are sequentially dependent, and losing any packet will render all the following packets in the entire bitstream useless, even though these packets are correctly received.

In this paper, we take an integrated approach $[18,19]$ toward joint source coding and error control by incorporating packetization and layered coding in the source coder and finding a new error-control strategy. Our approach applies equally to unicast and multicast. In the sequel, we base our exposition on multicast in general and RLM in particular as unicast is a special case of multicast.
We design a packetized wavelet video (PWV) coder based on the work in [20] that generates layered bitstreams that are independent among layers while being embedded within each layer. Packetization is simply done by rounding each layer of the bitstream to its nearest packet boundary. This was shown to suffer little source-coding performance loss in [21]. The PWV coder achieves better rate-distortion (R-D) performance than 3D SPIHT. In addition, and because different layers in the PWV bitstream are independent, a lost packet in the PWV bitstream only renders the following packets in the same layer useless. It will not affect packets in other layers, making it more error-robust than 3D SPIHT.

Layered channel coding is accomplished in our system using a systematic rate-compatible RS style erasure code [22]. The server multicasts all PWV bitstream layers and all parity layers to separate multicast groups. With error control, each receiver can decide to subscribe to or unsubscribe from the multicast groups based on the packet loss ratio and the available channel bandwidth, as determined by the congestion control mechanism $[11,12]$.

The layered structure of PWV calls for error-control strategies in video streaming that offer UEP not only among bitstream layers, but also within packets in each layer. It is this interplay between source coding and error control that distinguishes our integrated paradigm from past "plug-anduse" approaches. We formulate a rate-allocation problem and give a multi-layered error control solution in the form of optimal collection of multicast groups (or collection of source and channel layers) for the receiver to subscribe. This FEC-based error-control mechanism can be constructed as an extension of the approach in [17], which is single-layered in nature.

Using ideas of the "digital fountain" approach [23], we also consider pseudo-ARQ $[17,24]$ in the above FEC system for reliable multicast by sending delayed parity packets to some additional multicast groups at the server. Within a tolerable delay bound, the receiver is allowed to join and subsequently leave these groups to retrieve packets that are lost in previous transmissions. Error control in this FEC/pseudoARQ system in terms of a receiver joining and leaving multicast groups is given by the subscription policy in a finitehorizon Markov decision process [15].

While both the PWV coder and the multilayered errorcontrol strategy are new-the former incorporates embedded wavelet video coding and packetization and the latter extends the single-layered approach in [17] — the main contribution of this paper lies in the synergistic integration of the two. Theoretical analysis shows a gain of up to one $\mathrm{dB}$ on a channel with $20 \%$ packet loss using our combined approach over separate designs of the source coder and the error-control mechanism. This is also substantiated by our simulations with a gain of up to $0.6 \mathrm{~dB}$.

Recent work $[24,25,26]$ on multimedia streaming has shown the benefit of the joint design of the source coders and the streaming protocols. While our integrated approach echoes this interlayer interaction philosophy, we focus on combining the two most important components of Internet streaming video: source coding and error control 


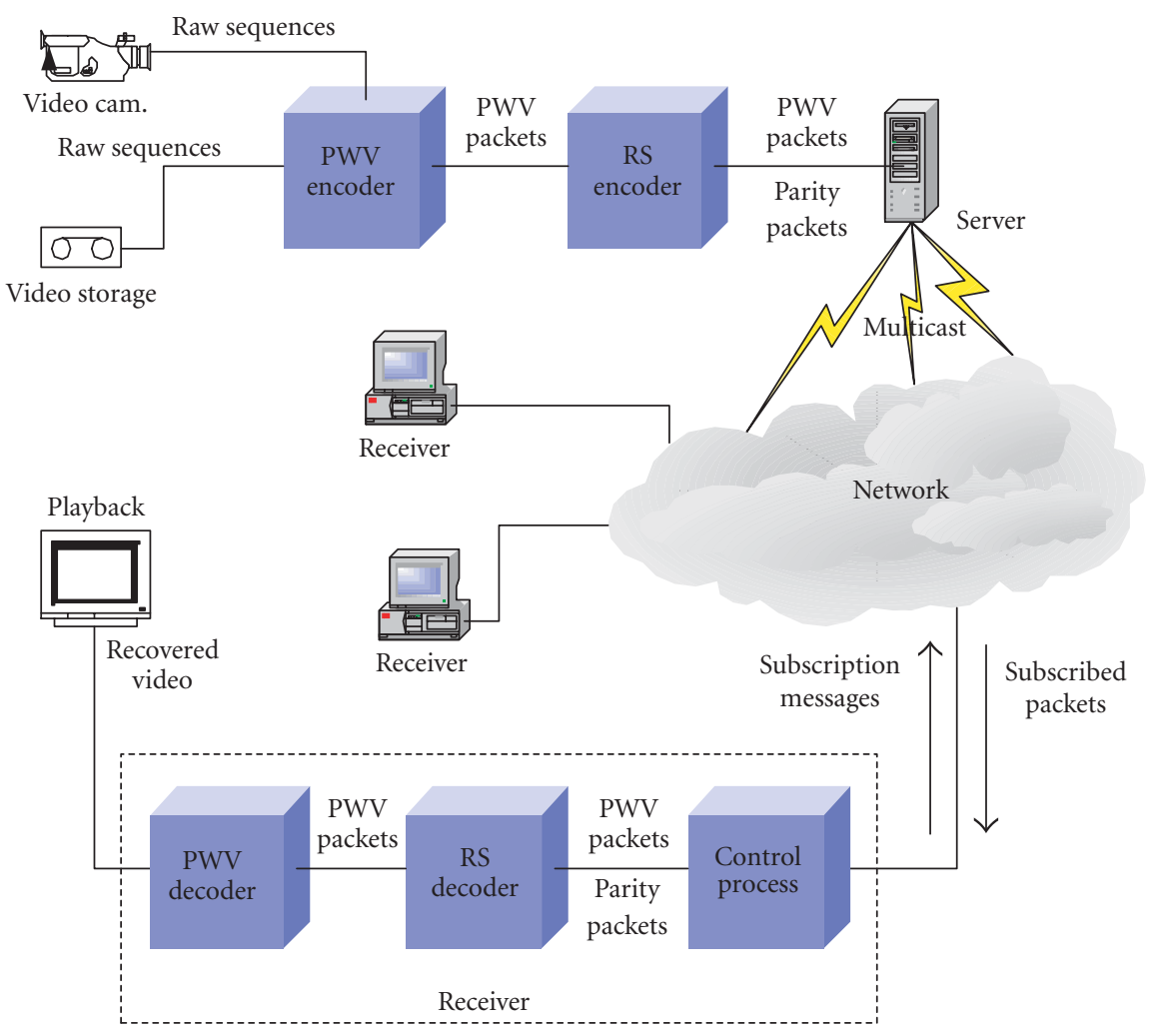

FIGURE 1: Block diagram of our integrated video multicast system.

(see Figure 1 for the block diagram of our system). We do not address congestion control $[11,12]$ in this work, although our system allows easy incorporation of TCP-friendly congestion-control protocols to form a true end-to-end architecture for video streaming. This opens doors for more exciting research and we leave this aspect of our work to future publications.

The rest of this paper is organized as follows. Section 2 focuses on our source-coding and packetization schemes that lead to the PWV coder. Section 3 describes our FECbased error-control model, while Section 4 presents combined PWV coding and FEC-based error control. Section 5 considers pseudo-ARQ and outlines the pseudo-ARQ-based error-control model. Section 6 presents combined PWV coding and FEC/pseudo-ARQ. Section 7 includes both analytical and simulation results. Section 8 concludes the paper.

\section{SOURCE CODING AND PACKETIZATION}

In this section, we describe our schemes for source coding and packetization, leading to the development of a PWV coder that facilitates easy integration with error control for Internet streaming.

\subsection{Source coding}

Although international standards like MPEG-2 [27] for video compression have been developed during the past decade for a number of important commercial applications (e.g., satellite TV and DVD), these algorithms cannot meet the general needs of Internet video because they are not designed or optimized for handling packet loss and heterogeneity in the emerging world of packet networks. Scalable coding, also known as layered, embedded, or progressive coding, is very desirable in Internet streaming because it encodes a video source in layers, like an onion, that facilitate easy bandwidth adaptation. But it is extremely difficult to write a compression algorithm that can layer the data properly, without a performance penalty. That is, a scalable compression algorithm inherently delivers lower quality than an algorithm that can optimally encode the source monolithically, like a solid ball. So, the difficulty lies in minimizing the effect of this structural constraint on the efficiency of the compression algorithm, both in terms of computational complexity and quality delivered at a given bandwidth.

Standard algorithms do not do well in this regard. Experiments with H.263+ in scalable mode show that, compared with monolithic (nonlayered) coding [28], the average PSNR loses roughly $1 \mathrm{~dB}$ with each layer. The main focus of the MPEG-4 standard [4] is object-based coding and the scalability in it is very limited. MPEG-4's streaming video profile on FGS coding [7] only provides flexible rate scalability and the coding performance is still about $1-1.5 \mathrm{~dB}$ lower than that of a monolithic coding scheme [29]. In addition, error propagation [30] due to packet loss is particularly severe if the video-coding scheme exploits temporal redundancy of the video sequence as H.263+ and MPEG-4. 
3D wavelet video coding $[31,32,33,34]$ deviates from the standard motion compensated DCT approach in H.263+ or MPEG-4. Instead, it seeks after alternative means of video coding by exploiting spatiotemporal redundancies via $3 \mathrm{D}$ wavelet transformation. Promising results have been reported. For example, Choi and Woods [34] presented better results than MPEG-1 using a 3D subband approach, together with hierarchical variable-size block-based motion compensation. In particular, the 3D SPIHT [6] video coder, which is a $3 \mathrm{D}$ extension of the celebrated SPIHT image coder [35], was chosen by Microsoft as the basis of its next-generation streaming video technology [17]. The latest 3D embedded wavelet video (3D EWV) coder [20], which borrows ideas from the 2D EBCOT algorithm [36], showed for the first time that $3 \mathrm{D}$ wavelet video coding outperforms MPEG-4 coding by as much as $2 \mathrm{~dB}$ for most low-motion and average-motion sequences. $3 \mathrm{D}$ EWV also has comparable performance to MPEG-4 for most high-motion sequences. In this work, we choose to use the 3D EWV coder because of its good performance and its embeddedness. In the following, we briefly review the 3D EWV coding algorithm.

The Daubechies 9/7 biorthogonal filters of [37] are used in all three dimensions to perform a separable wavelet decomposition in the 3D EWV coder. The temporal transform and $2 \mathrm{D}$ spatial transform are done separately by first performing a dyadic wavelet decomposition in the temporal direction, and then within each of the resulting temporal bands, performing three levels of a 2D spatial dyadic decomposition.

After 3D wavelet transformation, the wavelet coefficients can be coded with a bit-plane coding scheme like 3D SPIHT [6]. The 3D EWV algorithm is more powerful yet flexible than 3D SPIHT. It is powerful because the context formation in arithmetic coding does not have to be restricted to the rigid cubic structure imposed by zerotrees in 3D SPIHT. It is flexible due to the fact that samples on each bit-plane are coded one at a time, making the extension to object-based coding very easy. The core of the algorithm consists of the following three parts.

(1) 3D context modeling. Adaptive context formation in 3D EWV primarily relies on a binary-valued state variable $\sigma[i, j, k]$ that characterizes the significance ${ }^{1}$ of coefficient $x[i, j, k]$ at position $[i, j, k]$ after subband transposition. It is initialized to 0 and toggled to 1 when $x[i, j, k]$ 's first nonzero bit-plane value is encoded. Depending on the state of $\sigma[i, j, k]$, the binary information bit of $x[i, j, k]$ is coded at each bit-plane using one of the following three primitives: zero coding (ZC), sign coding (SC), and magnitude refinement (MR). If $\sigma[i, j, k]=0$ in the current bit-plane, ZC and $\mathrm{SC}$ are used to code new information about $x[i, j, k]$; otherwise, MR is used instead. Each of the above three coding primitives has its own context formation and assignment rules.

\footnotetext{
${ }^{1} \mathrm{~A}$ coefficient is called significant if it is nonzero at the current bit-plane level.
}
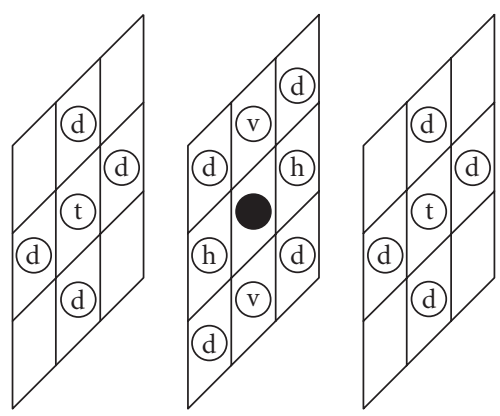

Current sample

(h) Horizontal neighbor

(V) Vertical neighbor

(t) Temporal neighbor

(d) Diagonal neighbor

FIGURE 2: Immediate neighbors are considered in context formation and assignments for ZC in 3D EWV.

(i) ZC. When a coefficient $x[i, j, k]$ is not yet significant in previous bit-planes, this primitive is used to code new information about whether it becomes significant or not in the current bit-plane. ZC uses significance information about $x[i, j, k]$ 's immediate neighbors as contexts to code its own significance information (see Figure 2).

(ii) SC. Once $x[i, j, k]$ becomes significant in the current bit-plane, the SC primitive is called to code its sign. SC also utilizes high-order context-based arithmetic coding with fourteen contexts.

(iii) MR. This primitive is used to code new information about $x[i, j, k]$ if it becomes significant in a previous bit-plane. MR uses three contexts for arithmetic coding.

(2) Fractional bit-plane coding. With the above three coding primitives in bit-plane coding, an embedded bitstream can be generated for each subband with excellent coding performance. The practical coding gain of 3D EWV over 3D SPIHT [6] (and EBCOT [36] over SPIHT [35]) stems from two aspects: one lies in highorder context modeling for SC and MR; the other one is the use of fractional bit-plane coding, which provides a practical means of scanning the wavelet coefficients within each bit-plane for R-D optimization at different rates. Specifically, the coding procedure in $3 \mathrm{D}$ EWV consists of three consecutive passes in each bit-plane.

(i) Significance propagation pass. This pass processes coefficients that are not yet significant but have a preferred neighborhood. A coefficient is designated as having a preferred neighborhood if and only if it has at least one significant immediate diagonal neighbor for diagonal bands, or at least one significant horizontal, vertical, or temporal neighbor for other bands. For these coefficients, the ZC primitive is used to code their significance information in the current bit-plane and, if any of them becomes significant in the current bit-plane, the SC primitive is used to compress their sign bits.

(ii) Magnitude refinement pass. Coefficients that became significant in previous bit-planes are coded 
in this pass. The binary bits corresponding to these coefficients in the current bit-plane are coded by the MR primitive.

(iii) Normalization pass. Processed in this pass are coefficients that were not coded in the previous two passes. These coefficients are not yet significant, so only ZC and SC are applied in this pass.

Each of the above passes processes one fractional bitplane in the natural raster scan order. Note that processing ZC and MR in different fractional bit-planes comes naturally from their separate treatments in context modeling. In addition, the processing order of the three fractional bit-planes follows the order of their perceived R-D significance levels. The first fractional bit-plane typically achieves a higher R-D ratio than the second one, which in turn is easier to code than the third one. Using fractional bit-plane coding thus ensures that each subband gives an R-D optimized embedded bitstream.

(3) Bitstream construction and scalability. In the previous coding stage of $3 \mathrm{D} \mathrm{EWV}$, an embedded bitstream is generated for each subband. In this stage, bitstreams corresponding to different subbands are truncated and multiplexed to construct a final bitstream. The question now is how to determine where to truncate a bitstream and how to multiplex different bitstreams in order to provide functionalities such as rate and resolution scalability. The bitstream truncation and multiplexing procedure is described as follows.

(i) Bitstream truncation with R-D optimization. Given a target bit rate $R_{0}$, our objective is to construct a final bitstream that satisfies the bit rate constraint and meanwhile minimizes the overall distortion. The end of each fractional bit-plane is a candidate truncation point. The R-D pair at each candidate truncation point can be obtained by calculating the bitstream length and distortion at that point. An operational R-D curve can be constructed for each subband. All valid truncation points must lie on the convex hull of the R-D curve to guarantee R-D optimality at each truncation point. Optimal rate allocation over all subbands is achieved when operation points on all operational R-D curves have an equal slope $\lambda$. The slope $\lambda_{0}$, corresponding to $R_{0}$, is found via a fast bisectional algorithm [38].

(ii) Multilayer bitstream construction. To make an $L$ layer bitstream, $L$ R-D slopes $\lambda_{1}, \lambda_{2}, \ldots, \lambda_{L}$ with $\left|\lambda_{1}\right|>\left|\lambda_{2}\right|>\cdots>\left|\lambda_{L}\right|$ are first chosen. A corresponding truncation point (hence a layer of bitstream) is found from each subband for every R-D slope $\lambda_{i}$. The corresponding layers from all the subbands constitute the $i$ th layer of the final bitstream. Depending on its available bandwidth and computational capability, the receiver can selectively decode the first few layers.

(iii) Bitstream scalability. Fractional bit-plane coding in 3D EWV ensures that the final bitstream is scalable with fine granularity. Furthermore, the final bitstream can be rearranged to achieve other functionalities easily because the offset and length of each layer of bitstream for each subband are coded in the header of the bitstream. This makes the final bitstream very flexible for use in applications like video browsing and multicasting over the Internet.

\subsection{Packetization}

In the above original EWV coder, bitstream truncation at the end of each fractional bit-plane (i.e., treating each fractional bit-plane as a basic unit in bitstream formation) makes sense because each bit spent in coding a fractional bit-plane reduces the distortion by roughly the same amount. In addition, multiplexing different layers according to the decreasing magnitudes of their R-D slopes gives the best progressive coding performance. These strategies work great in terms of improving source-coding performance. But they might not be suitable for designing source coders for video streaming applications that involve JSCC, in which it often pays to leave some redundancy in the source bitstream. Thus, our philosophy in packetization is to achieve bitstream resynchronization and easy integration with error control via judicious modification of the original EWV coder so that the sacrifice in source-coding performance is small.

Note that packetizing the original EWV bitstream into fixed-length packets, in general, is not allowed, because the truncation points in the EWV bitstream typically are not set on packet boundaries. This is due to the fact that the EWV bitstream is not as fine-grained as the 3D SPIHT bitstream, which can be truncated at the byte level, making fixed-length packetization trivial as in [17].

To rectify this shortcoming in the original EWV bitstream, we mark any multiple of packet size in the bitstream (instead of the end of each fractional bit-plane) as a candidate truncation point in EWV coding. In addition, we skip the bitstream multiplexing step and output multiple layered bitstreams in the new video coder for the purpose of increasing error resilience. In forming each bitstream layer, we note that the original 3D EWV coder already provides lots of flexibilities-it allows a multilayered structure with each layer corresponding to one or several subbands and also achieves spatial/temporal scalability by coding each group of subbands independently into an embedded bitstream. In this work, we form layers by resolution, that is, we choose to encode all the subbands in each resolution into an embedded bitstream for each layer. See Figure 3 for a 2D example.

The bitstream layers allow R-D truncation at each layer for a given target bit rate (typically given in terms of the number of packets per GOF). Because of the constraint that candidate truncation points for each bitstream layer must lie on packet boundaries, optimal rate allocation over all layers is achieved when the slopes at operation points on all operational R-D curves are approximately equal.

We now have a new coder that generates packetized layered bitstreams with each layer having an integer number of 


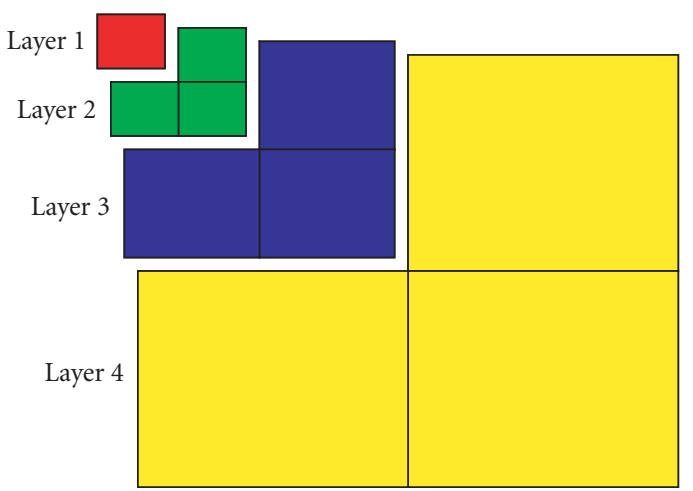

Figure 3: A 2D example where all the subbands in each resolution are coded into an embedded bitstream for each layer.

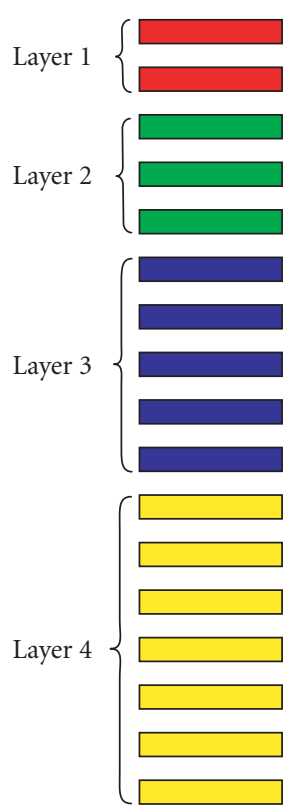

GOF 1

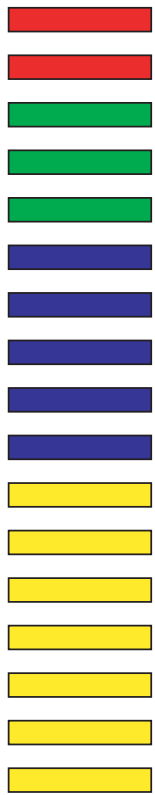

GOF 2

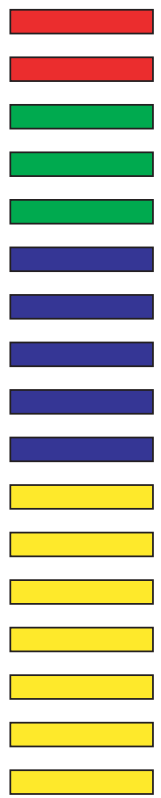

GOF $K$
FIgURE 4: The PWV coder generates packetized layered bitstreams for each GOF that are independent among layers while being embedded within each layer.

packets and being embedded (see Figure 4). We call it a 3D PWV coder. For example, when a color QCIF sequence is coded at 50 packets (1000 bytes per packet) per GOF of 32 frames using a three-level wavelet transform, the lengths of the four bitstream layers are typically $2,6,17$, and 25 packets. Each layer of the PWV bitstream can be independently decoded, thus error resilience is improved during transmission when compared with transmitting the original EWV bitstream.

It was shown in [21] that the source coding performance of the PWV coder is very close to that of the original EWV coder. That is, the performance loss due to packetization is very small when there is no packet loss. To see this, the PWV

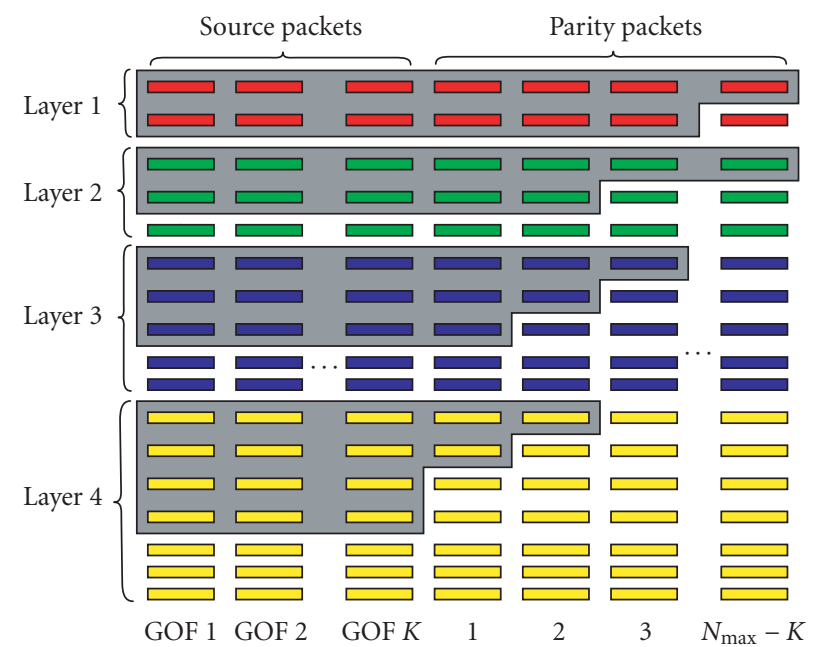

FIgURE 5: The $\left(N_{\max }, K\right)$ rate-compatible RS erasure code is used to generate $N_{\max }-K$ parity packets for each $K$-packet coding block. A typical receiver subscribes to source/parity packets highlighted in shaded areas.

bitstream can be thought of as a slightly modified version of the original EWV bitstream after rounding each EWV bitstream layer to its nearest packet boundary by either pruning the extra fractional packet or growing it out to fill the remaining fractional packet. Of course, the EWV bitstream formation involves multiplexing or interleaving of different bitstream layers whereas there is no such a step in PWV coding. In summary, the PWV coder achieves a performance close to EWV with very low complexity using a simple packetization scheme.

\section{FEC-BASED ERROR-CONTROL MODEL}

We now discuss our FEC-based error-control model. As we can see from Figure 4, the PWV bitstream in each layer is divided and packetized into a certain number of packets; packets from different GOFs along the horizontal (or time) axis form a sublayer. We partition each sublayer into coding blocks, each having $K$ source packets. The blocksize $K$ is constant across all sublayers. For each coding block, we apply a systematic $\left(N_{\max }, K\right)$ RS style erasure correction code [22] to produce $N_{\max }-K$ parity packets (see Figure 5 ). And $N_{\max }-K$ is the maximum amount of redundancy that will be needed by the transmitter to protect the source layer. It is determined by the worst channel condition. The $N_{\max }-K$ parity packets $\mathbf{p}_{1}, \ldots, \mathbf{p}_{N_{\max }-K}$ are generated byte-wise from the $K$ source packets $\mathbf{s}_{1}, \ldots, \mathbf{s}_{K}$ in the coding block by

$$
\left[\mathbf{s}_{1} \cdots \mathbf{s}_{K} \mid \mathbf{p}_{1} \cdots \mathbf{p}_{N_{\max }-K}\right]=\left[\mathbf{s}_{1} \cdots \mathbf{s}_{K}\right] G,
$$

where

$$
G=\left[I_{K} \mid P_{K, N_{\max }-K}\right]
$$

is the generator matrix over the finite Galois field $\mathrm{GF}\left(2^{8}\right)$ that is composed of a $K \times K$ identity matrix and a $K \times\left(N_{\max }-K\right)$ 


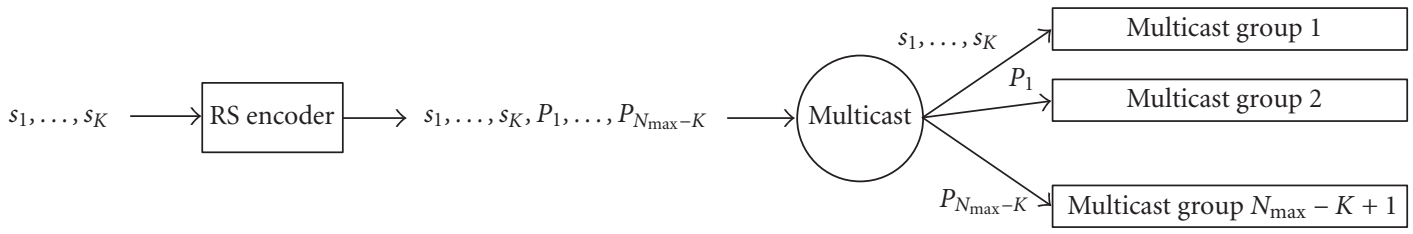

Figure 6: A coding block in any sublayer is first encoded to generate $N_{\max }-K$ parity packets, then the resulting $N_{\max }$ source plus parity packets are sent to $N_{\max }-K+1$ multicast groups.

parity generation matrix. The erasure code possesses the property that a minimum of $K$ source/parity packets suffice to recover the $K$ source packets.

In our RLM system with FEC, the transmitter buffers frames as they arrive. When a GOF is accumulated, it generates a PWV bitstream for this GOF. After PWV bitstreams are generated for $K$ GOFs, the transmitter computes the $N_{\max }-K$ parity packets for each coding block of $K$ source packets. The $K$ source packets are broadcasted to one multicast group, while the $N_{\max }-K$ parity packets are broadcasting to $N_{\max }-K$ multicast groups. This is illustrated in Figure 6 . Thus, a coding block uses $N_{\max }-K+1$ multicast groups in total. Note that this scheme, also used in $[17,39]$, is different from traditional FEC schemes [40] that broadcast all parity packets to one multicast group. It avoids overwhelming the network with unduly heavy load from unwanted parity packets.

According to the current network condition (e.g., packet loss ratio and available bandwidth), for each coding block a receiver makes its own decision in terms of which multicast groups of that block to subscribe. The receiver can subscribe to no multicast group at all, to the first multicast group only at low latency, or to the first multicast group plus any number of multicast groups for improved video quality but at high latency. This allows the receiver to trade latency for quality - another advantage of the multicast structure of Figure 6. Source/parity packets in the shaded areas in Figure 5 indicate those the receiver subscribes to in different coding blocks. Note that unsubscribed packets typically reside at the bottom of each bitstream layer as they are less important in the R-D sense. This corresponds to UEP strategies not only among PWV bitstream layers, but also among packets within each layer-a scenario that is markedly different from the case considered in [17].

From the received source/parity packets, the receiver instantly recovers as many source packets as possible and decode them. As long as the total number of correctly received packets in an RS coded block is greater than or equal to $K$, all the $K$ source packets can be recovered. Playback begins after $K$ GOFs are decoded. Thus the delay is the duration of $K$ GOFs.

Due to the fact that the tolerable coding delay is limited for streaming video, the length $K$ in a channel-coding block should be small. For example, $K=8$ corresponds to a coding delay of roughly eight seconds if the GOF size is 32 . For such a small value of $K$, the RS erasure code is a good choice because of its maximal erasure correction capability [22] and low complexity.

\section{COMBINED PWV CODING AND FEC-BASED ERROR CONTROL}

In this Section, we assume that a congestion-control mechanism (e.g., AIMD [11] and TCP-friendly RAP [12]) is available and formulate the multilayered error-control problem under a fixed transmission rate and packet loss ratio.

\subsection{Problem formulation}

To facilitate easy intergration of PWV coding and FEC-based error control, PWV bitstreams for different GOFs are generated so that the number of layers and the number of packets/sublayers within each layer are fixed. Suppose that the PWV bitstream for each GOF has $L$ layers with $P_{l}$ packets in the $l$ th layer. Assume that the receiver subscribes to a total of $N_{l, i}$ source plus parity packets per coding block in sublayer $\{l, i\}$ (which is the $i$ th sublayer of the $l$ th layer). These packets are highlighted in the shaded areas in Figure 5.

Define $\mathbf{N}=\left(N_{1,1}, \ldots, N_{1, P_{1}}, \ldots, N_{L, 1}, \ldots, N_{L, P_{L}}\right)$ as the rate allocation vector. It specifies the rate allocation between source packets and parity packets within each source sublayer. The total transmission rate, in terms of packets per GOF, is given by

$$
R(\mathbf{N})=\sum_{l=1}^{L} \sum_{i=1}^{P_{l}} \frac{N_{l, i}}{K}, \quad N_{l, i} \in\left\{0, K, K+1, \ldots, N_{\max }\right\} .
$$

From Section 3, we have $N_{l, i}=0$ or $K \leq N_{l, i} \leq N_{\max }$. When $N_{l, i}>K$, the factor $N_{l, i} / K$ measures the redundancy which the receiver chooses in order to protect source packets in sublayer $\{l, i\}$. If the packet losses are independent with probability $\epsilon$, then, after channel decoding, any packet in that sublayer can be recovered with probability

$$
\begin{aligned}
P_{l, i}\left(N_{l, i}\right)= & \frac{1}{K} \sum_{s=0}^{K} \sum_{c=0}^{N_{l, i}-K}\left(\begin{array}{c}
K \\
s
\end{array}\right) \epsilon^{K-s}(1-\epsilon)^{s} \\
& \times\left(\begin{array}{c}
N_{l, i}-K \\
c
\end{array}\right) \epsilon^{N_{l, i}-K-c}(1-\epsilon)^{c} \\
& \times \begin{cases}K, & \text { if } s+c \geq K, \\
s, & \text { if } s+c<K .\end{cases}
\end{aligned}
$$


The double sum computes the expected number of correctly recovered source packets within one coding block in sublayer $\{l, i\}$. Note that when $s+c \geq K$, all the $K$ source packets can be recovered. While if $s+c<K$, this number can only be $s$, no matter how many parity packets are received. The expected reconstruction distortion per GOF is given by

$$
D(\mathbf{N})=D_{0}-\sum_{l=1}^{L} \sum_{i=1}^{P_{l}} P_{l, \leq i} \Delta D_{l, i}
$$

where

$$
\begin{aligned}
P_{l, \leq i}= & P(\text { the first } i \text { sublayers in the } l \text { th layer } \\
& \quad \text { are decoded correctly }) \\
= & \prod_{i^{\prime}=1}^{i} P_{l, i^{\prime}}\left(N_{l, i^{\prime}}\right)
\end{aligned}
$$

$D_{0}$ is the expected reconstruction distortion when the transmission rate is zero, and $\Delta D_{l, i}$ represents the expected reduction of distortion if the packet in sublayer $\{l, i\}$ can be decoded. Let $D_{l, 0}$ be the expected reconstruction distortion of the $l$ th layer when the transmission rate is zero, then we have $D_{0}=\sum_{l=1}^{L} D_{l, 0}$.

In our analysis, we use an operational distortion-rate function $D(R)=\sigma^{2} 2^{-2 R / A}$ to model the R-D performance of the PWV coder, where $A$ is a scaling factor. Based on this model, $\Delta D_{l, i}$ can be computed from $D_{l, 0}$ as $\Delta D_{l, i}=$ $D_{l, 0}\left(2^{-2 i / A_{l}}-2^{-2(i-1) / A_{l}}\right)$. Because each layer of the PWV bitstream is embedded, a packet is dependent on those ahead of it within the same layer. That the $i$ th packet can be decoded implies that the $i-1$ packets ahead of it can also be correctly decoded; this dependency is reflected in (6).

Equations (3) and (5) give the total transmission rate and the expected distortion as a function of the rate allocation vector $\mathbf{N}$. Now we want to find the optimal rate allocation vector to minimize the expected distortion subject to a transmission rate constraint. That is, we consider the following constraint optimization problem:

$$
\min _{\mathbf{N}} D(\mathbf{N}) \text { subject to } R(\mathbf{N}) \leq R_{0},
$$

where $R_{0}$ is the given rate constraint.

\subsection{The optimization algorithm}

One way to solve the above problem is by finding the rate allocation vector $\mathbf{N}$ that minimizes the Lagrangian

$$
\begin{aligned}
J(\mathbf{N})= & D(\mathbf{N})+\lambda R(\mathbf{N}) \\
= & D_{0}-\sum_{l=1}^{L} \sum_{i=1}^{P_{l}}\left(\prod_{i^{\prime}=1}^{i} P_{l, i^{\prime}}\left(N_{l, i^{\prime}}\right)\right) \Delta D_{l, i} \\
& +\lambda \sum_{l=1}^{L} \sum_{i=1}^{P_{l}} \frac{N_{l, i}}{K} .
\end{aligned}
$$

The solution to this problem is completely characterized by the set of distortion increments $\Delta D_{l, i}$, which are determined by the source coding and packetization, and the probability $P_{l, i^{\prime}}\left(N_{l, i^{\prime}}\right)$ with which a packet in sublayer $\{l, i\}$ can be correctly recovered, which is in turn determined by the channel coding. There are many methods to solve this optimization problem with a rate constraint $[13,14,15]$.

We solve this problem by using an iterative approach that is based on the method of alternating variables for multivariable minimization [41]. The objective function

$$
J(\mathbf{N})=J\left(N_{1,1}, \ldots, N_{1, P_{1}}, \ldots, N_{L, 1}, \ldots, N_{L, P_{L}}\right)
$$

in (8) is minimized over one variable at a time, while keeping the other variables constant, until convergence. To be specific, let $\mathbf{N}^{(0)}$ be the initial rate allocation vector. Let $\mathbf{N}^{(t)}=$ $\left(N_{1,1}^{(t)}, \ldots, N_{L, P_{L}}^{(t)}\right)$ be determined for $t=1,2, \ldots$, as follows. Select one component $N_{l_{t}, i_{t}} \in\left\{N_{1,1}, \ldots, N_{L, P_{L}}\right\}$ to optimize at step $t$. This can be done in a round-robin style. Then for $N_{l, i}\left(l \neq l_{t}\right.$ or $\left.i \neq i_{t}\right)$, let $N_{l, i}^{(t)}=N_{l, i}^{(t-1)}$. For $N_{l_{t}, i_{t}}$, we perform the following rate optimization:

$$
\begin{aligned}
N_{l_{t}, i_{t}}^{(t)} & =\arg \min _{N_{l_{t}, i_{t}}} J\left(N_{1,1}, \ldots, N_{1, P_{1}}, \ldots, N_{L, 1}, \ldots, N_{L, P_{L}}\right) \\
& =\arg \min _{N_{l_{t}, i_{t}}} \sum_{i=i_{t}}^{P_{l_{t}}}\left(-\prod_{i^{\prime}=1}^{i} P_{l_{t}, i^{\prime}}\left(N_{l_{t}, i^{\prime}}\right)\right) \Delta D_{l_{t}, i}+\lambda \frac{N_{l, i_{t}}}{K} .
\end{aligned}
$$

Equation (11) contains only those items in (8) that are related to $N_{l_{t}, i_{t}}$. For fixed $\lambda$, the 1-dimensional minimization problem (11) can be solved using standard nonlinear optimization procedures, such as gradient-descent-type algorithm [41]. Now, in order to minimize the Lagrangian $J(\mathbf{N})$ given by (8), we proceed as follows: first, for fixed $\lambda$, we minimize $J(\mathbf{N}, \lambda)$ and get a total transmission rate $R(\mathbf{N}, \lambda)$. Compare this rate with the target transmission rate and accordingly adjust $\lambda$. This procedure is repeated until convergence. Generally, the resulting $R(\mathbf{N})$ will not be exactly equal to the target rate constraint, because it only picks limited discrete values.

In our experiments, we always start with the initial rate allocation vector $\mathbf{N}=(1,1, \ldots, 1)$. We cycle through all the components, beginning with the component associated with the first sublayer and ending with the component associated with the last sublayer. The resulting rate allocation

$$
\mathbf{N}^{*}=\left(N_{1,1}^{*}, \ldots, N_{1, P_{1}}^{*}, \ldots, N_{L, 1}^{*}, \ldots, N_{L, P_{L}}^{*}\right)
$$

gives the optimal error-control solution, generally in the form of unequal error protection, for the different source sublayers.

\section{PSEUDO-ARQ-BASED ERROR-CONTROL MODEL}

In this Section, we augment the FEC-based error-control model by considering (ARQ), which is extensively used in packet networks because it makes the most use of the network capacity. In conventional ARQ, only those packets lost during previous transmissions are retransmitted. Obviously, it is adaptive because the number of retransmission requests reflects exactly the current packet loss probability. However, ARQ is regarded as impractical in multicast because of the 
feedback implosion problem. As a common approach, feedback suppression partially solves this problem at the expense of increased latency, more complexity at receivers, or additional requirements of the network.

Nonnenmacher et al. [40] demonstrate that hybrid FEC/ARQ is very powerful in reducing the number of retransmissions at low packet loss rate. But it cannot completely eliminate the need of retransmissions, especially when the number of receivers grows large or the packet loss rate becomes high. Byers et al. [23] further develop this idea by using pure FEC to form a digital fountain for reliable multicast of bulk data. It can be viewed as a form of pseudo-ARQ. Application of hybrid FEC/Pseudo-ARQ to video multicast is studied in $[17,24]$. ARQ is simulated by sending delayed parity packets to some additional multicast groups. The receiver can join and subsequently leave these groups to retrieve packets that are lost in previous transmissions. This scheme can satisfy the retransmission needs of a large number of receivers with a small number of retransmitted parity packets.

In our work, we also applied this hybrid method on the PWV encoded video for multicast. Specifically, instead of being transmitted at the same time of the source packets, some of the parity packets are multicast in subsequent time slots with different delays. According to the current number of received packets, the network condition, and the available transmission rate, each receiver can choose to join these multicast groups to retrieve the delayed parity packets. Figure 7 depicts the flowgraph of our pseudo-ARQ-based error-control scheme. Obviously, this pseudo-ARQ scheme is more efficient than pure FEC because the delayed parity packets are subscribed to only when necessary. However, this efficiency is at the expense of larger latency. In real applications, the tolerable latency is limited and the number of decision time slots should also be fixed. Thus we have a problem of making optimal subscription decisions at different time slots in order to minimize the expected reconstruction distortion under a transmission rate constraint.

\section{COMBINED PWV CODING AND FEC/PSEUDO-ARQ}

Just as in pure FEC, the goal of error control in hybrid $\mathrm{FEC/pseudo-ARQ}$ is to minimize the expected distortion in the reconstruction given a transmission rate constraint. However, with ARQ the receiver can take a series of actions based on the state of each step. This control process at each receiver can be modeled as a finite horizon Markov decision process [15]. A Markov decision process with finite horizon $W$ is a $W$-step stochastic process through a state space. An action is associated with each trellis state to maximize or minimize an expected quantity. The assignment of actions to trellis states is called a policy.

In this problem, each state $s$ in the trellis space is uniquely determined by the number of received source packets $x$, the number of total received packets $k$, and the step number (or time index) $w$. We want the policy $\pi$ to minimize $J(\pi)=$ $D(\boldsymbol{\pi})+\lambda R(\boldsymbol{\pi})$. This can be solved by a dynamic programming algorithm, which regressively minimizes the partial

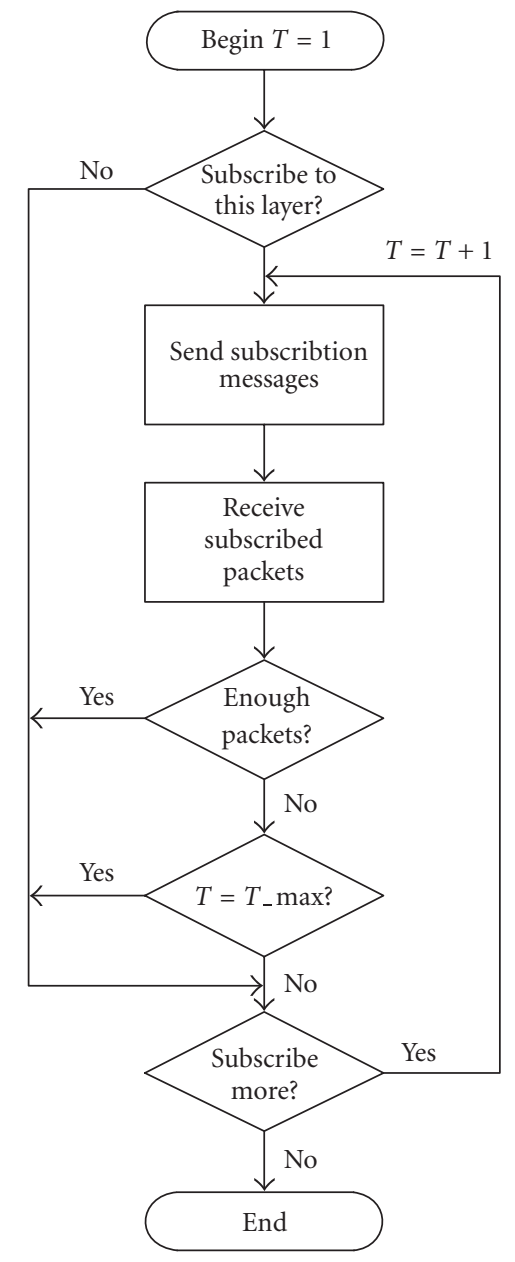

FIgURE 7: The decision process at each receiver for any sublayer. $T \_$max is the number of decision time slots.

Lagrangian $\tilde{J}(\pi, s)$ of each state $s$ as a function of the transition probability to the next state $s^{\prime}$

$$
\widetilde{J}(\pi, s)=\sum_{s^{\prime}} P\left(s^{\prime} \mid s, \pi(s)\right) \times\left[\Delta \widetilde{J}\left(s^{\prime} \mid s, \pi(s)\right)+\widetilde{J}\left(\pi, s^{\prime}\right)\right] .
$$

Here, the partial Lagrangian $\widetilde{J}(\pi, s)$ represents the cost $D+\lambda R$ beginning from state $s$ with policy $\pi ; P\left(s^{\prime} \mid s, \pi(s)\right)$ is the transition probability from the current state $s$ to the next state $s^{\prime}$ given the policy component $\pi(s)$ at state $s ; \Delta \tilde{J}\left(s^{\prime} \mid s, \pi(s)\right)$ represents the cost reduction in this transition and it is not related to states other than $s$ and $s^{\prime}$. At state $s$, the algorithm updates $\tilde{J}(\pi, s)$ and $\pi(s)$, respectively, by

$$
\begin{aligned}
\tilde{J}\left(\pi^{*}, s\right)= & \min _{\pi^{*}(s)} \sum_{s^{\prime}} P\left(s^{\prime} \mid s, \pi^{*}(s)\right) \\
& \times\left[\Delta \widetilde{J}\left(s^{\prime} \mid s, \pi^{*}(s)\right)+\widetilde{J}\left(\pi^{*}, s^{\prime}\right)\right], \\
\pi^{*}(s)= & \arg \min _{\pi^{*}(s)} \sum_{s^{\prime}} P\left(s^{\prime} \mid s, \pi^{*}(s)\right) \\
& \times\left[\Delta \widetilde{J}\left(s^{\prime} \mid s, \pi^{*}(s)\right)+\widetilde{J}\left(\pi^{*}, s^{\prime}\right)\right] .
\end{aligned}
$$


Let $L_{\text {total }}=\sum_{l} P_{l}$ represent the total number of sublayers; let $W$ be the number of decision steps and $K$ the size of an RS coding block. The algorithm runs as follows.

Algorithm 1. (1) Initialize the policy components $\pi(l, s)$ of all the layers $0 \leq l<L_{\text {total }}$ and all the states $s(0 \leq w<$ $W, 0 \leq k \leq K, 0 \leq x \leq k)$ throughout the trellis space; set $\lambda$ to an initial value.

(2) Set $\pi_{\text {old }}(l, s)=\pi(l, s)$ for all $l$ and $s$.

(3) Start from the first layer $l=0$.

(4) Start from the last step $w=W-1$; set $J_{\text {next }}(l, s)=0$ for all $l$ and $s$.

(5) For each state s, compute $J(l, s)$ which represents the cost $D+\lambda R$ of the current layer $l$ starting from the current step $w$, given the current policy $\pi$ and $J_{\text {next }}(l, s)$ which represents the cost starting from the next step $w+1$.

(6) Find the optimal policy component $\pi^{*}(1, s)(15)$ for all the states $s$ at the current step $w$ which minimize $J(l, s)$. $\operatorname{Set} J(l, s)$ to the new minimum and $\pi(l, s)=\pi^{*}(l, s)$.

(7) Let $w=w-1$; if $w \geq 0$, set $J_{\text {next }}(l, s)=J(l, s)$ for all $l$ and $s$ and go to step (5).

(8) Let $l=l+1$; if $l<L_{\text {total }}$, go to step (4).

(9) If $\pi(l, s)=\pi_{\text {old }}(l, s)$ for all $l$ and $s$, convergence is met for the current $\lambda$; else go to step (2).

(10) Compute the expected transmission rate $R$ with the current policy $\pi$.

(11) Check if the computed $R$ has most closely approached the

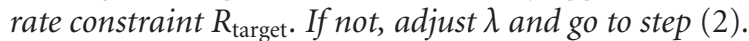

(12) End.

\section{RESULTS}

\subsection{Analysis}

The optimization algorithm described in Section 4.2 extends the single-layered optimization algorithm in [17]. To compare the performance difference between the two errorcontrol mechanisms, we assume that bitstreams generated by 3D SPIHT and PWV coding have the same R-D curve, that is, $D(R)=\sigma^{2} 2^{-2 R / A}$. Note that the 3D SPIHT bitstream has a sequential dependency among all the source packets of a GOF, whereas in the PWV coder only packets within the same layer are sequentially dependent.

Applying the algorithm described in Sections 4 and 6, we compute the signal-to-reconstruction noise ratio as a function of the transmission rate as shown in Figure 8 for $\epsilon=$ $20 \%$ and in Figure 9 for $\epsilon=5 \%$. We assume that the PWV bitstream consists of four layers, with the numbers of packets in different layers being 2, 6, 17, and 25 .

When only pure FEC is used, we see that integrating PWV coding and error control outperforms the singlelayered approach in [17] by up to one $\mathrm{dB}$ when $\epsilon=20 \%$ and up to $0.6 \mathrm{~dB}$ when $\epsilon=5 \%$. In the hybrid FEC/pseudoARQ case, however, the difference of performance between the two coders becomes very small. Note that when ARQ is introduced, any subscribed packet can almost always reach the receiver with little increase in the expected transmission

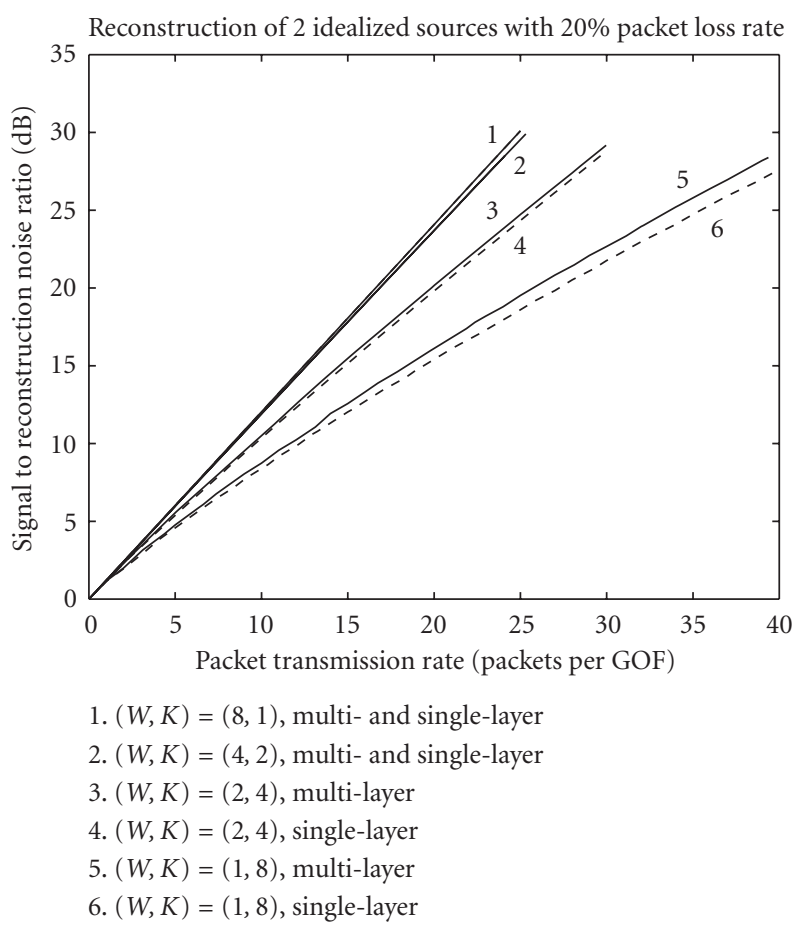

FIGURE 8: Analytical results using optimal error control for transmitting a single-layered video bitstream and a multilayered video bitstream over a network with $20 \%$ packet loss.

rate. Thus, the dependency among layers does not play a significant role any more. Take $(W, K)=(8,1)$, for example, if we fix the policy component $\pi(s=0, c=0, w=0)=1$ and increase the policy components $\pi(s=0, c=0,0<w<8)$ from 0 to 1 , the expected transmission rate is increased by only 0.25 (from 1 to 1.25 ) packets per GOF. However, now the probability that any packet in the corresponding layer cannot be recovered is reduced from $20 \%$ to $3 \times 10^{-6}$. Also note that in Figure 9 for $\epsilon=5 \%$, because the packet loss rate is small enough, both $(W, K)=(8,1)$ and $(W, K)=(4,2)$ can achieve near-perfect transmission. Therefore, they actually share the same performance curve.

\subsection{Simulations}

Simulations are also carried out, in which two 288-frame 25 fps QCIF color sequences Foreman and Akiyo, encoded using the PWV coder and protected with a systematic RS erasure code with a block size $K=8$, are transmitted over a simulated network with $20 \%$ and $5 \%$ packet loss, respectively. Each video sequence is blocked into 9 GOFs containing 32 frames per GOF and encoded at 50 packets per GOF with 1000 bytes per packet. The duration of each GOF is 1.28 seconds. Thus, the number of packets $N$ allowed for each GOF in term of the transmission rate $R$ in bps is given by $N=1.28 R / 8000$ packets per GOF.

The quantities $\Delta D_{l, i}$ needed for the solution in Section 4 are given by the encoded source packets and revealed to the receiver. In real applications, they can be either sent by the 


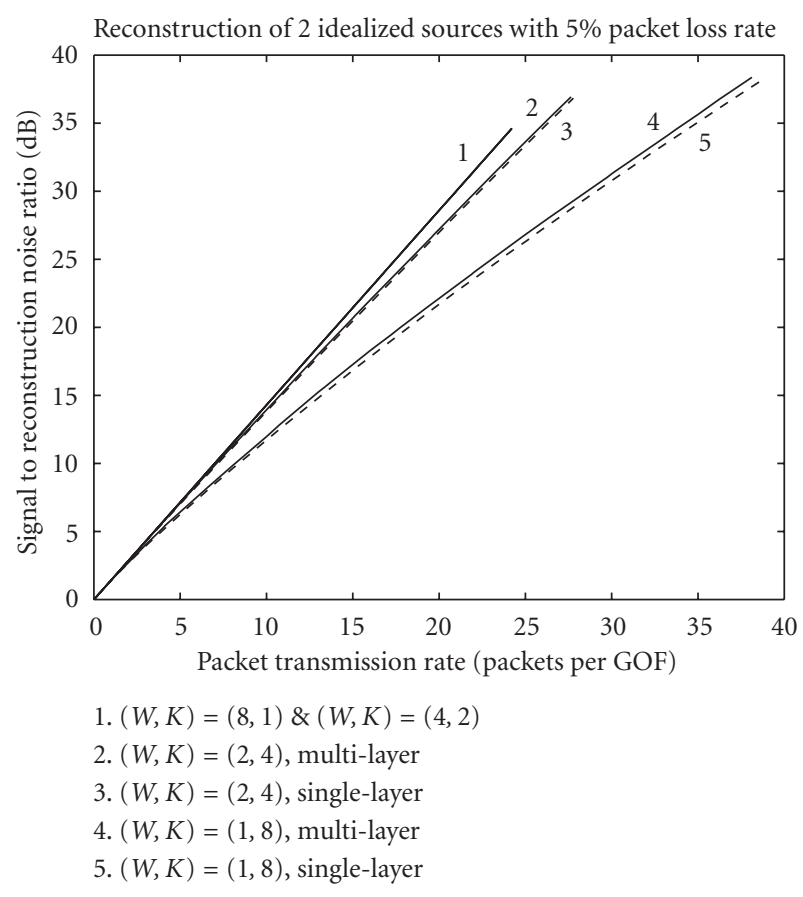

Figure 9: Analytical results using optimal error control for transmitting a single-layered video bitstream and a multilayered video bitstream over a network with $5 \%$ packet loss.

server as a side information or estimated adaptively by the receiver using previously recovered packets. For each transmission rate, the selected combination of source and parity packets is transmitted and each simulation runs 100 times.

In order to compare our multilayered scheme with the single-layered scheme in [17] on a fair basis, we modify the PWV coder to make it produce single-layered bitstreams. That is, the packets of each GOF have a sequential dependency as in 3D SPIHT. Loss of any packet will render the following packets of the same GOF useless. Thus we have a single-layered version of the PWV coder with comparable coding efficiency to the original PWV coder. The same procedure as in [17] is applied to provide error protection for the bitstreams generated by this new coder. In Figures 10, 11, 12, and 13 , we refer to the single-layered PWV coder as SPWV and the multilayered PWV coder as MPWV.

Figure 10 presents the average PSNRs of two sets of simulations using Foreman based on the two versions of PWV, respectively. The packet loss rate is $20 \%$. Note that when pure FEC is used, the multilayered approach gains up to $0.64 \mathrm{~dB}$. This gap widens as the number of subscribed source packets goes larger. When $W=2$, the maximum gain reduces to $0.3 \mathrm{~dB}$. When $W=4,8$, there is virtually no difference between the two because the error-control strategy is strong enough to ensure every subscribed packet to be correctly recovered.

Figure 11 shows the same results as in Figure 10, but with a different packet loss rate of $5 \%$. The maximum performance difference is about $0.5 \mathrm{~dB}$, which happens in pure

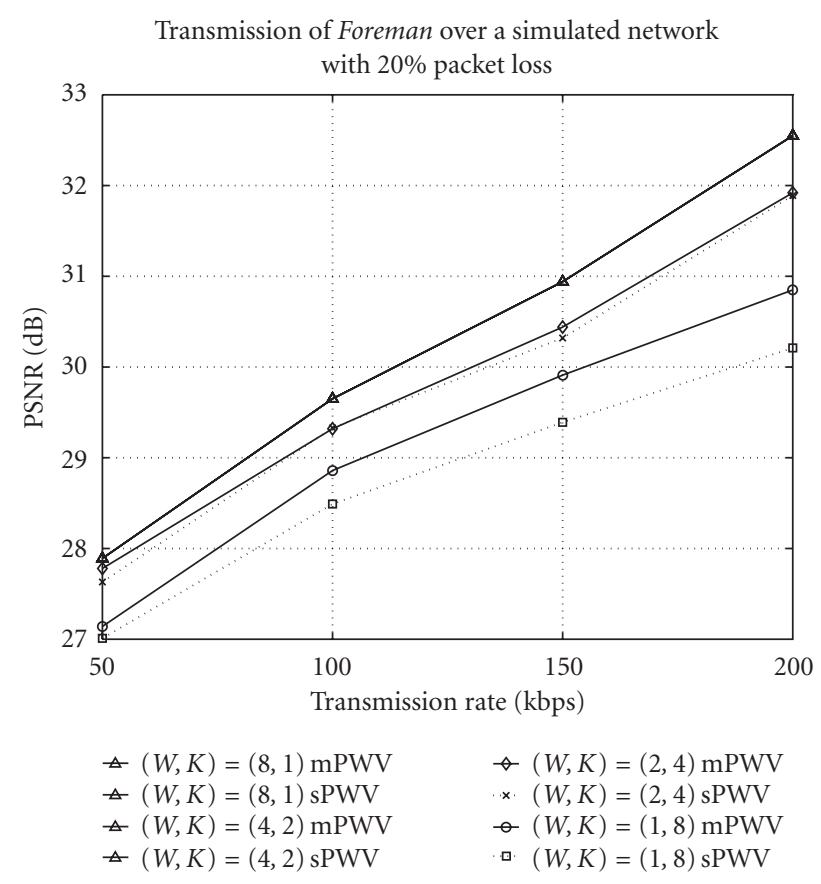

FIGURE 10: Simulation results using error control for transmitting mPWV and sPWV coded Foreman over a network with $20 \%$ packet loss.

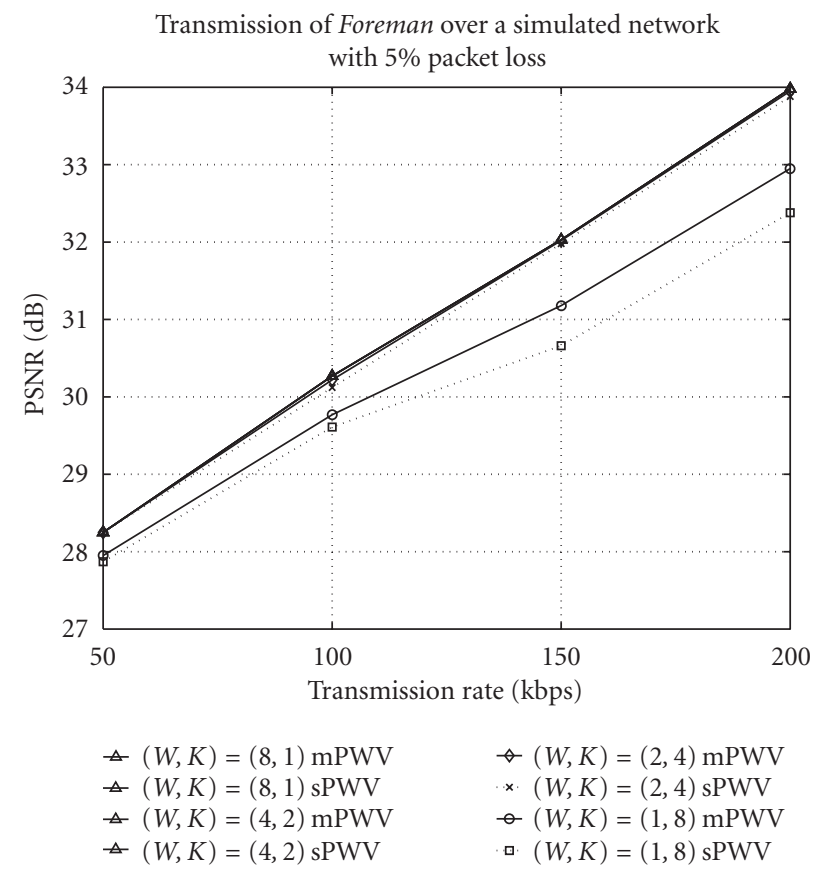

FIGURE 11: Simulation results using error control for transmitting mPWV and sPWV coded Foreman over a network with 5\% packet loss.

FEC case at the highest rate. With a small packet loss rate of $5 \%$, hybrid FEC/pseudo-ARQ method with $W=2$ already 


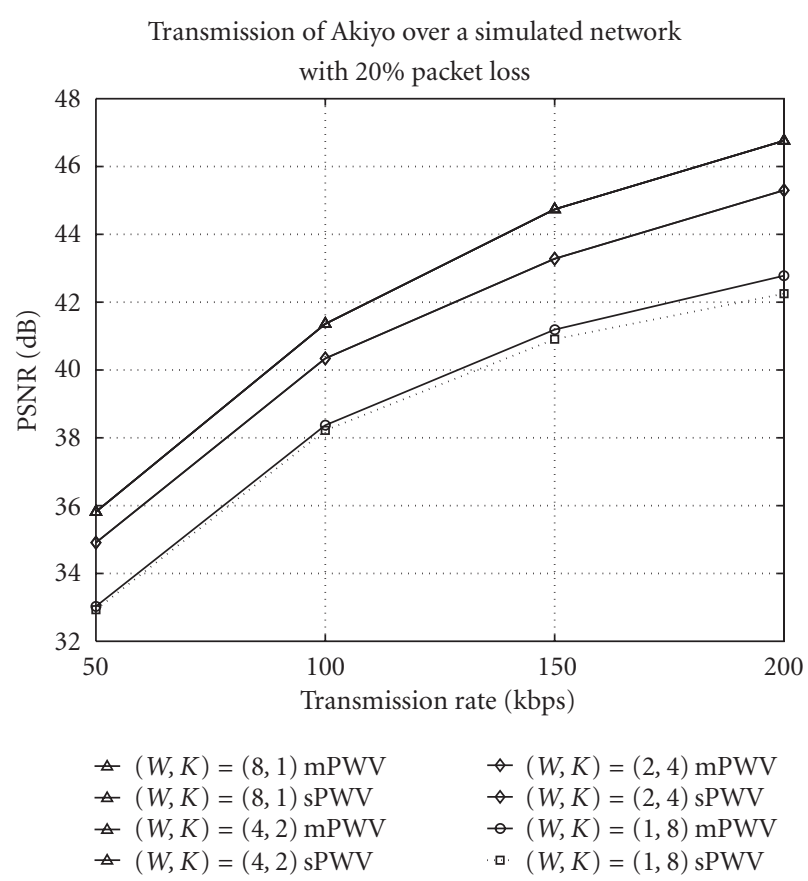

FIGURE 12: Simulation results using error control for transmitting mPWV and sPWV coded Akiyo over a network with $20 \%$ packet loss.

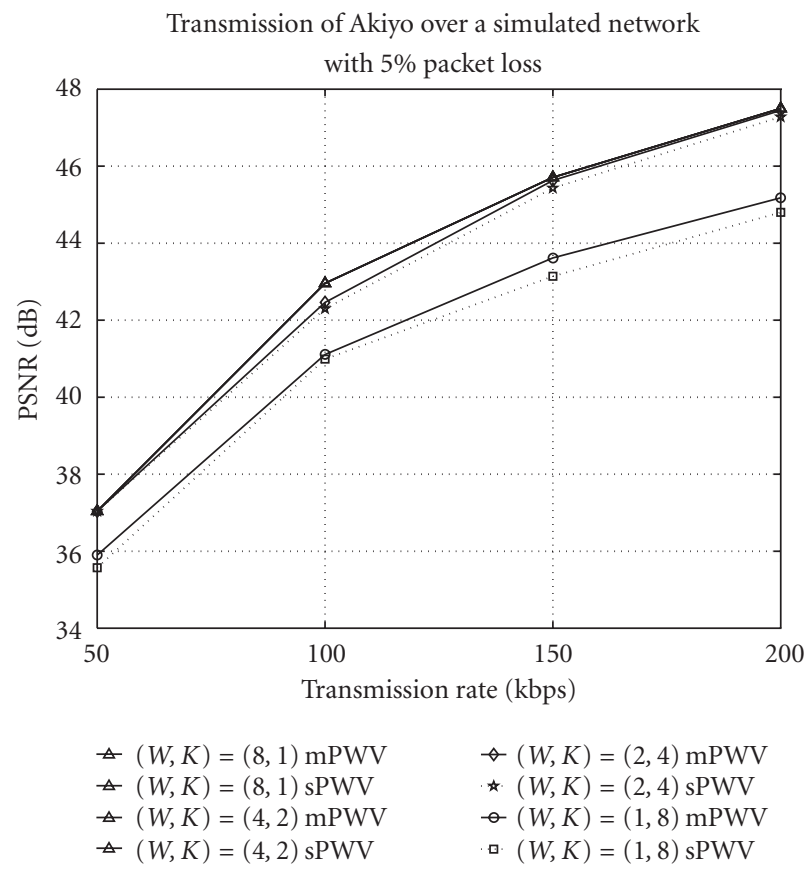

FIGURE 13: Simulation results using error control for transmitting $\mathrm{mPWV}$ and sPWV coded Akiyo over a network with 5\% packet loss.

achieves performance very close to higher $W=4$ and $W=$ 8 cases. This substantiates our analysis result as shown in Figure 9.
TABLE 1: Numbers of subscribed source packets of all four layers in the transmission of PWV coded Foreman using pure FEC with a packet loss rate of $20 \%$ at four different transmission rates.

\begin{tabular}{lcrrr}
\hline$R_{\text {total }}($ kbps $)$ & 50 & 100 & 150 & 200 \\
\hline$P_{\text {total }}($ packets $)$ & 8 & 16 & 24 & 32 \\
$S_{1}$ (packets) & 1 & 1 & 1 & 2 \\
$S_{2}$ (packets) & 2 & 3 & 3 & 4 \\
$S_{3}$ (packets) & 1 & 4 & 7 & 9 \\
$S_{4}$ (packets) & 1 & 2 & 4 & 5 \\
$S_{\text {total }}$ (packets) & 5 & 10 & 15 & 20 \\
\hline
\end{tabular}

TABle 2: Numbers of subscribed source packets of all four layers in the transmission of PWV coded Foreman using hybrid FEC/pseudoARQ $((W, K)=(2,4))$ with a packet loss rate of $20 \%$ at four different transmission rates.

\begin{tabular}{lcrrr}
\hline$R_{\text {total }}($ kbps $)$ & 50 & 100 & 150 & 200 \\
\hline$P_{\text {total }}$ (packets) & 8 & 16 & 24 & 32 \\
$S_{1}$ (packets) & 1 & 1 & 1 & 2 \\
$S_{2}$ (packets) & 2 & 3 & 3 & 4 \\
$S_{3}$ (packets) & 2 & 5 & 8 & 9 \\
$S_{4}$ (packets) & 1 & 2 & 5 & 6 \\
$S_{\text {total }}$ (packets) & 6 & 11 & 17 & 21 \\
\hline
\end{tabular}

The simulation results using Akiyo are presented in Figure 12 for 20\% packet loss rate and in Figure 13 for 5\% packet loss rate, respectively. Average PSNRs of two sets of simulations based on single-layered PWV coding and multilayered PWV coding are presented. The maximal gain in pure FEC is $0.53 \mathrm{~dB}$ for $20 \%$ packet loss rate and 0.48 for $5 \%$ packet loss rate. Because the Akiyo sequence contains much less high-frequency components than Foreman, packets in higher-frequency layers have a smaller contribution to the reduction of distortion. As a result when pseudo-ARQ is used ( $W=2,4,8)$, the subscription policy tends to spend more on channel coding part instead of increasing the source rate. Thus, there is very little packet loss in pseudo-ARQ cases, and the performance of the two error-control schemes are almost the same.

Table 1 lists the number of subscribed source packets of all four layers in the transmission of PWV coded Foreman using pure FEC with a packet loss rate of $20 \%$. Note that $R_{\text {total }}$ is the total transmission rate in kbps. And $P_{\text {total }}$ is the corresponding number of 1000-byte packets at rate $R_{\text {total }}$. The number of subscribed source packets of the ith layer is denoted by $S_{i}(i=1,2,3,4)$. These numbers are determined by the UEP algorithm described in Section 4. From Table 1, we see that the source bitstreams at lower transmission rates (e.g., 50, 100, and $150 \mathrm{kbps}$ ) are truncated versions of one preencoded embedded bitstream at a higher rate (e.g., $200 \mathrm{kbps}$ ). Reencoding is thus avoided, and progressive transmission in video streaming or receiver adaptation in video multicast is made possible. 


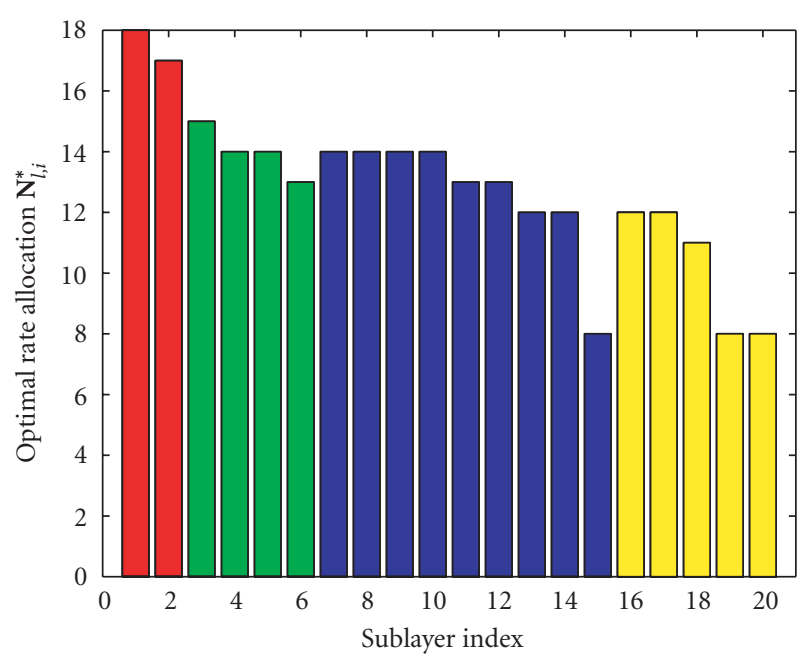

Figure 14: Rate allocation $\mathbf{N}^{*}$ in pure FEC when the transmission rate is $200 \mathrm{kbps}$ and the packet loss ratio is $20 \%$.

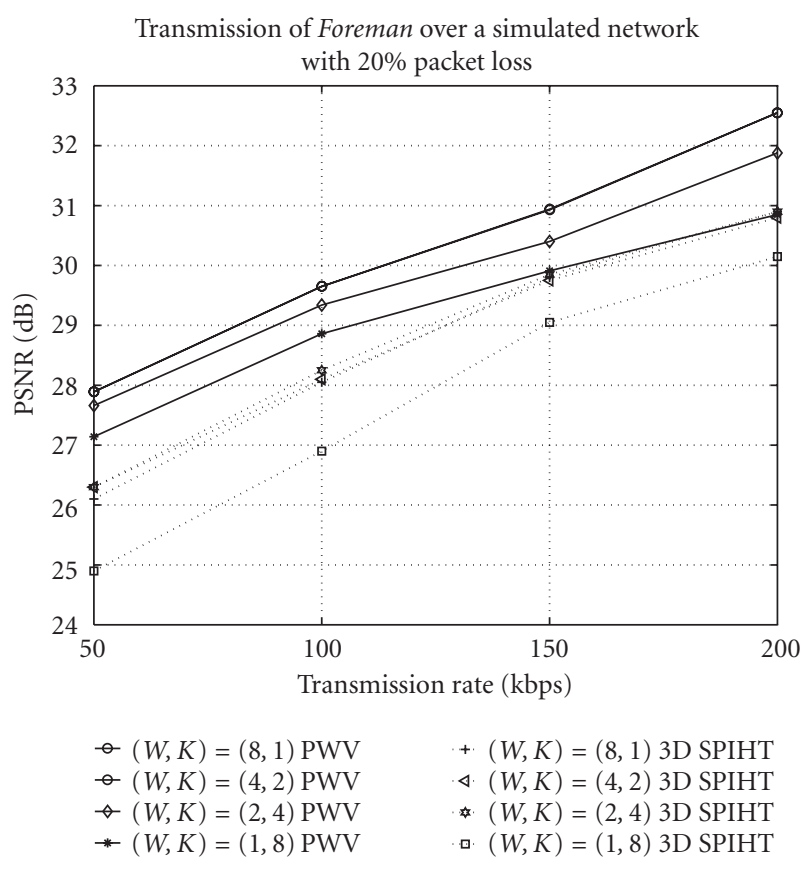

FIGURE 15: Simulation results using error control for transmitting PWV and 3D SPIHT coded Foreman over a network with $20 \%$ packet loss.

Table 2 depicts the number of subscribed source packets of all four layers in the transmission of PWV coded Foreman using hybrid FEC/pseudo-ARQ $((W, K)=(2,4))$ with a packet loss rate of $20 \%$. Although the FEC/pseudo-ARQ solution degenerates to the FEC solution when $W=1$ (e.g., no ARQ); when $W>1$, a receiver is allowed to subscribe to more source packets, taking advantage of hybrid FEC/pseudo-ARQ. The difference in $S_{3}$ and $S_{4}$ between results in the two tables highlights this point.
Figure 14 depicts the rate allocation $\mathbf{N}^{*}$ used in our pure FEC simulations of Foreman when the transmission rate is $200 \mathrm{kbps}$ (or 32 packets per GOF). A receiver subscribes to a total of 20 source sublayers and 256 source/parity packets for $K=8$ GOFs in this case.

Finally, we also compare the performance difference between our PWV-based error-control strategy and the approach in [17] using 3D SPIHT. The average PSNRs in simulations of Foreman are computed and plotted in Figure 15 for $20 \%$ packet loss rate at four different transmission rates.

Results of pure FEC, as a special case of hybrid FEC/Pseudo-ARQ when $W=1$, shows that our multilayered scheme using PWV outperforms the single-layered approach in [17] using 3D SPIHT with a gain up to $2.2 \mathrm{~dB}$. In the case of hybrid FEC/Pseudo-ARQ, there is also a corresponding gain of up to $1.6 \mathrm{~dB}$, which mainly comes from the higherefficient encoding. The gap between the hybrid method and pure FEC is between 0.8 and $1.8 \mathrm{~dB}$.

\section{SUMMARY}

We present an integrated approach toward combined source coding and error-control design for RLM of video based on the PWV source coder and RS erasure channel codes. Both analysis and simulations show gains of our integrated framework over previous work. The practical gain stems from the fact that the PWV source coder achieves better R-D performance than 3D SPIHT and that our new multilayered error control mechanism based on the PWV bitstream is superior to the single-layered one in [17].

In this paper, we assume that a separate congestion control mechanism is carried out at each receiver to determine the available bandwidth in RLM. Further work incorporating quality adaptation into our combined source coding and error control framework would be desirable. We also assume that packet loss is random in the network, which does not hold in many situations. Designing error control for transmitting video over networks with burst packet loss should be also considered as a future work.

\section{ACKNOWLEDGMENTS}

The authors gratefully acknowledge Jianping Hua's assistance in the PWV coder design. This work supported by the National Science Foundation (NSF) Career grant MIP-0096070, the NSF grant CCR-01-04834, the Army Research Office (ARO) YIP grant DAAD19-00-1-0509, and the Office of Naval Research (ONR) YIP grant N00014-01-1-0531.

\section{REFERENCES}

[1] "Special issue on real-time video over the Internet," Signal Processing: Image Communication, vol. 15, September 1999.

[2] "Special issue on multimedia over IP," IEEE Trans. Multimedia, vol. 3, March 2001.

[3] "Special issue on streaming video," IEEE Trans. Circuits and Systems for Video Technology, vol. 11, March 2001. 
[4] ISO/IEC JTC 1/SC29/WG11 N2687, "MPEG-4 Video Verification Model Version 13.0," March 1999.

[5] ITU-T Recommendation H.263, version 2, "Video coding for low bitrate communication," January 1998.

[6] B.-J. Kim, Z. Xiong, and W. A. Pearlman, "Low bit-rate scalable video coding with $3 \mathrm{D}$ set partitioning in hierarchical trees (3D SPIHT)," IEEE Trans. Circuits and Systems for Video Technology, vol. 10, no. 8, pp. 1365-1374, 2000.

[7] H. Radha, M. van der Schaar, and Y. Chen, "The MPEG4 fine-grained scalable video coding method for multimedia streaming over IP," IEEE Trans. Multimedia, vol. 3, no. 1, pp. 53-68, 2001.

[8] S. Paul, Multicasting on the Internet and Its Applications, Kluwer Academic, Boston, Mass, USA, 1998.

[9] S. Servetto and K. Nahrstedt, "Broadcast quality video over IP,” IEEE Trans. Multimedia, vol. 3, no. 1, pp. 162-173, 2001.

[10] S. R. McCanne, Scalable compression and transmission of Internet multicast video, Ph.D. thesis, University of California, Berkeley, Calif, USA, December 1996.

[11] D. Chiu and R. Jain, "Analysis of the increase and decrease algorithms for congestion avoidance in computer networks," Journal of Computer Networks and ISDN, vol. 17, no. 1, pp. 1-14, 1989.

[12] R. Rejaie, M. Handley, and D. Estrin, "Layered quality adaptation for Internet video streaming," IEEE Journal on Selected Areas in Communications, vol. 18, no. 12, pp. 2530$2544,2000$.

[13] J. Lu, A. Nosratinia, and B. Aazhang, "Progressive sourcechannel coding of images over bursty error channels," in Proc. International Conference on Image Processing, Chicago, Ill, USA, October 1998.

[14] M. Ruf and J. Modestino, "Operational rate-distortion performance for joint source and channel coding of images," IEEE Trans. Image Processing, vol. 8, no. 3, pp. 305-320, 1999.

[15] V. Chande, H. Jafarkhani, and N. Farvardin, "Joint sourcechannel coding of images for channels with feedback," in Proc. IEEE Information Theory Workshop, San Diego, Calif, USA, February 1998.

[16] J. Hagenauer, "Rate-compatible punctured convolutional codes (RCPC codes) and their applications," IEEE Trans. Communications, vol. 36, no. 4, pp. 389-400, 1988.

[17] P. A. Chou, A. E. Mohr, A. Wang, and S. Mehrotra, "Error control for receiver-driven layered multicast of audio and video," IEEE Trans. Multimedia, vol. 3, no. 1, pp. 108-122, 2001.

[18] T. Chu and Z. Xiong, "Combined wavelet video coding and error control for Internet streaming and multicast," in Proc. GlobeCom '01, San Antonio, Tex, USA, November 2001.

[19] T. Chu, J. Hua, and Z. Xiong, "Packetized wavelet video coding and error control for receiver-driven layered multicast: an integrated approach," in Proc. Multimedia Signal Processing Workshop, Cannes, France, October 2001.

[20] J. Hua, Z. Xiong, and X. Wu, "High-performance 3-D embedded wavelet video (EWV) coding," in Proc. Multimedia Signal Processing Workshop, Cannes, France, October 2001.

[21] X. Wu, S. Cheng, and Z. Xiong, "On packetization of embedded multimedia bitstreams," IEEE Trans. Multimedia, vol. 3, no. 1, pp. 132-140, 2001.

[22] L. Rizzo, "Effective erasure codes for reliable computer communication protocols," ACM Computer Communication Review, vol. 27, no. 2, pp. 24-36, 1997.

[23] J. Byers, M. Luby, M. Mitzenmacher, and A. Rege, "A digital fountain approach to reliable distribution of bulk data," in Proc. ACM SIGCOMM '98, pp. 56-67, Vancouver, BC, Canada, September 1998.

[24] W.-T. Tan and A. Zakhor, "Real-time Internet video using error resilient scalable compression and TCP-friendly transport protocol," IEEE Trans. Multimedia, vol. 1, no. 2, pp. 172-186, 1999.

[25] S. Servetto and K. Nahrstedt, "Video streaming over the public Internet: Multiple description codes and adaptive transport protocols," in Proc. IEEE International Conference on Image Processing, vol. 3, Kobe, Japan, October 1999.

[26] R. Puri, K. Lee, K. Ramchandran, and V. Bharghavan, "An integrated source transcoding and congestion control paradigm for video streaming in the Internet," IEEE Trans. Multimedia, vol. 3, no. 1, pp. 18-32, 2001.

[27] B. G. Haskell, A. Puri, and A. N. Netravali, Digital Video: An Introduction to MPEG-2, Chapman \& Hall, New York, NY, USA, 1997.

[28] L. Yang, F. C. M. Martins, and T. R. Gardos, "Improving H.263+ scalability performance for very low bit rate applications," in Proc. Visual Communications and Image Processing, San Jose, Calif, USA, January 1999.

[29] S. Li, F. Wu, and Y.-Q. Zhang, "Study of a new approach to improve FGS video coding efficiency," in ISO/IEC MPEG 50th meeting, M5583, Maui, Hawaii, USA, December 1999.

[30] E. Steinbach, N. Farber, and B. Girod, "Standard compatible extension of H.263 for robust video transmission in mobile environments," IEEE Trans. Circuits and Systems for Video Technology, vol. 7, no. 6, pp. 872-881, 1997.

[31] G. Karlsson and M. Vetterli, "Three dimensional subband coding of video," in Proc. IEEE Int. Conf. Acoustics, Speech, Signal Processing, pp. 1100-1103, New York, NY, USA, April 1988.

[32] D. Taubman and A. Zakhor, "Multirate 3-D subband coding of video," IEEE Trans. Image Processing, vol. 3, no. 5, pp. 572$588,1994$.

[33] J. R. Ohm, "Three-dimensional subband coding with motion compensation," IEEE Trans. Image Processing, vol. 3, no. 5, pp. 559-571, 1994.

[34] S. J. Choi and J. W. Woods, "Motion compensated 3-D subband coding of video," IEEE Trans. Image Processing, vol. 8, no. 2, pp. 155-167, 1999.

[35] A. Said and W. A. Pearlman, "A new, fast, and efficient image codec based on set partitioning in hierarchical trees," IEEE Trans. Circuits and Systems for Video Technology, vol. 6, no. 3, pp. 243-250, 1996.

[36] D. Taubman, "High performance scalable image compression with EBCOT," IEEE Trans. Image Processing, vol. 9, no. 7, pp. 1158-1170, 2000.

[37] M. Antonini, M. Barlaud, P. Mathieu, and I. Daubechies, "Image coding using wavelet transform," IEEE Trans. Image Processing, vol. 1, no. 2, pp. 205-220, 1992.

[38] Y. Shoham and A. Gersho, "Efficient bit allocation for an arbitrary set of quantizers," IEEE Trans. Acoustics, Speech, and Signal Processing, vol. 36, no. 9, pp. 1445-1453, 1988.

[39] W.-T. Tan and A. Zakhor, "Video multicast using layered FEC and scalable compression," IEEE Trans. Circuits and Systems for Video Technology, vol. 11, no. 3, pp. 373-386, 2001.

[40] J. Nonnenmacher, E. Biersack, and D. Towsley, "Parity-based loss recovery for reliable multicast transmission," IEEE Trans. Networking, vol. 6, no. 4, pp. 349-361, 1998.

[41] R. Fletcher, Practical Methods of Optimization, John Wiley \& Sons, New York, NY, USA, 2nd edition, 1987. 
Tianli Chu received the B.Eng. degree in telecommunication and information systems from Tsinghua University, China, in 1999, and the M.S. degree in electrical engineering in 2002 from Texas A\&M University, College Station, where he is currently working toward the Ph.D. degree in the Department of Electrical and Computer Engineering. His research interests are image and video coding, multimedia communication, and information theory.

Zixiang Xiong received the Ph.D. degree in electrical engineering in 1996 from the University of Illinois at Urbana-Champaign. From 1997 to 1999, he was with the University of Hawaii. Since 1999, he has been with the Department of Electrical Engineering at Texas A\&M University, where he is an Associate Professor. He spent the summers of 1998 and 1999 at Microsoft Research, Redmond, WA, and the summers of 2000 and

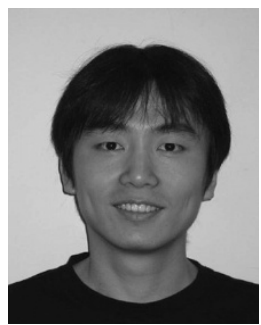
2001 at Microsoft Research in Beijing. His current research interests are Internet/wireless multimedia, distributed signal processing, and genomic image processing. Dr. Xiong received an NSF Career Award in 1999, an ARO Young Investigator Award in 2000, and an ONR Young Investigator Award in 2001. He also received an Eugene Webb Faculty Fellow Award in 2001 and a Select Young Faculty Award in 2002, both from Texas A\&M University. He is currently an Associate Editor for the IEEE Trans. on Circuits and Systems for Video Technology, the IEEE Trans. on Signal Processing, and the IEEE Trans. on Image Processing. 